\title{
Silencing of human polycomb target genes is associated with methylation of histone H3 Lys 27
}

\author{
Antonis Kirmizis, ${ }^{1}$ Stephanie M. Bartley, ${ }^{1}$ Andrei Kuzmichev, ${ }^{2}$ Raphael Margueron, ${ }^{2}$ \\ Danny Reinberg, ${ }^{2}$ Roland Green, ${ }^{3}$ and Peggy J. Farnham ${ }^{1,4}$ \\ ${ }^{1}$ McArdle Laboratory for Cancer Research, University of Wisconsin Medical School, Madison, Wisconsin 53706, USA; \\ ${ }^{2}$ Howard Hughes Medical Institute, Division of Nucleic Acids Enzymology, Department of Biochemistry, Robert Wood \\ Johnson Medical School, Piscataway, New Jersey 08854, USA; ${ }^{3}$ NimbleGen Systems Inc., Madison, Wisconsin 53711, USA
}

Polycomb group (PcG) complexes 2 and 3 are involved in transcriptional silencing. These complexes contain a histone lysine methyltransferase (HKMT) activity that targets different lysine residues on histones $\mathrm{H} 1$ or $\mathrm{H} 3$ in vitro. However, it is not known if these histones are methylation targets in vivo because the human PRC2/3 complexes have not been studied in the context of a natural promoter because of the lack of known target genes. Here we report the use of RNA expression arrays and CpG-island DNA arrays to identify and characterize human PRC2/3 target genes. Using oligonucleotide arrays, we first identified a cohort of genes whose expression changes upon siRNA-mediated removal of Suz12, a core component of PRC2/3, from colon cancer cells. To determine which of the putative target genes are directly bound by Suz12 and to precisely map the binding of Suz12 to those promoters, we combined a high-resolution chromatin immunoprecipitation (ChIP) analysis with custom oligonucleotide promoter arrays. We next identified additional putative Suz12 target genes by using ChIP coupled to CpG-island microarrays. We showed that HKMT-Ezh2 and Eed, two other components of the PRC2/3 complexes, colocalize to the target promoters with Suz12. Importantly, recruitment of Suz12, Ezh2 and Eed to target promoters coincides with methylation of histone H3 on Lys 27.

[Keywords: Suz12; histone methylation; polycomb; Eed; RNA interference; chromatin immunoprecipitation]

Supplemental material is available at http://www.genesdev.org.

Received March 3, 2004; revised version accepted April 27, 2004.

The Polycomb Group (PcG) proteins are negative regulators of transcription that were initially discovered in Drosophila (for review, see Jacobs and van Lohuizen 1999; Simon 2003). The PcG proteins, in conjunction with their positively acting counterparts known as Trithorax Group (TrxG) proteins, can maintain heritable transcription patterns of the homeotic (Hox) genes during development and differentiation (for review, see Jacobs and van Lohuizen 1999; Orlando 2003). Accordingly, genetic studies demonstrated that mutations in PcG proteins result in flies having transformed body segments because of inappropriate expression of Hox genes (Jurgens 1985). In addition to regulating developmental decisions, mammalian PcG proteins have been implicated in hematopoiesis, X-chromosome inactivation, and control of cell proliferation (for review, see Jacobs and van Lohuizen 2002). PcG proteins do not function

${ }^{4}$ Corresponding author.

E-MAIL farnham@oncology.wisc.edu; FAX (608) 262-2824.

Article and publication are at http://www.genesdev.org/cgi/doi/10.1101/ gad.1200204. alone but, instead, are assembled into multimeric complexes. The first PcG complex to be biochemically purified was named Polycomb repressive complex 1 (PRC1) and contains the PcG proteins polycomb (PC), polyhomeotic (PH), posterior sex comb (PSC), and dRING, among other polypeptides (Shao et al. 1999). Recent studies have biochemically defined another Drosophila polycomb complex, known as PRC2 or ESC-E(Z), whose core subunits are the PcG proteins Extra sex combs (Esc), Enhancer of zeste [E(z)], and Suppressor of zeste 12 [Su(z)12] and the histone-binding protein NURF55 (Czermin et al. 2002; Muller et al. 2002; Tie et al. 2003). Complexes similar to Drosophila PRC2 have also been purified from mammalian cells and consist of the human PcG proteins embryonic ectoderm development (Eed), the HKMTEzh2, Suz12, and the histone-binding proteins RbAp46 and 48 (Cao et al. 2002; Kuzmichev et al. 2002). Interestingly, four different forms of Eed exist in mammalian cells, and all can interact with Ezh2 and Suz12 resulting in distinct PRC complexes known as PRC2 and PRC3 (Kuzmichev et al. 2004; Pasini et al. 2004).

The PcG complexes have been proposed to control gene activity via transcriptional repression. Recently, in 
vitro assays have provided insight concerning the mechanism underlying PcG-mediated transcriptional repression. Characterization of the PRC2/3 complexes indicates that each complex contains an intrinsic histone lysine methyltransferase (HKMT) activity that is mediated by the SET [Su-(var)3-9;E(z);Trithorax] domain of Ezh2 (Kuzmichev et al. 2002). In vitro, the Ezh2 protein within the PRC2/3 complexes can methylate Lys 9 (H3K9) and Lys 27 (H3-K27) of histone H3 and Lys 26 of histone $\mathrm{H} 1$ (H1-K26), depending on whether the oligonucleosomes contain histone $\mathrm{H} 1$ (Kuzmichev et al. 2004). The efficiency with which the different histones (H1 or $\mathrm{H} 3$ ) are methylated depends on the specific form of Eed present in the complex (Kuzmichev et al. 2004). The PRC2 complex contains the longest form of Eed (Eed1) and methylates both H1-K26 and H3-K27. However, PRC2 preferentially methylates H1-K26 when nucleosome arrays contain histone $\mathrm{H} 1$. The PRC3 complex, which contains the two shortest forms of Eed (Eed3/4), methylates H3-K27. The intermediate form of Eed (Eed2) is also present in yet another distinct PRC complex (A. Kirmizis, R. Margueron, A. Kuzmichev, P. Farnham, and D. Reinberg, unpubl.). Based on these and previous discoveries, a model for the mechanism of PcGmediated transcriptional silencing has been proposed. The PRC2 or PRC3 complex is thought to first catalyze the addition of methyl groups to $\mathrm{H} 3-\mathrm{K} 27$ that serve as signals for the recruitment of PRC1. Binding of PRC1 is then proposed to either block the recruitment of transcriptional activating factors, such as SWI/SNF (a Trithorax complex), facilitating the establishment of a stable, repressive chromatin structure or to prevent transcription initiation by prebound factors (Simon 2003; Dellino et al. 2004).

Although an attractive model, it has not yet been demonstrated that the recruitment of HKMT-containing PRC complexes results in histone modification in mammalian cells owing to the fact that no target genes for these complexes have been identified. In contrast, target genes for PcG complexes have been identified in Drosophila. In the fruit fly, PcG target genes are regulated by specific DNA regions called Polycomb response elements (PREs; Simon et al. 1993). PREs are $2-3$ kb long and are composed of multiple different DNA motifs (Bloyer et al. 2003). Usually PREs contain binding sites for GAGA and PHO, two site-specific DNA-binding proteins that interact with PcG proteins (Poux et al. 2001) and function to recruit Drosophila PcG complexes to DNA (for review, see Pirrotta et al. 2003). Mammalian PREs have not yet been identified, and therefore, it remains unclear how mammalian PcG complexes are recruited to chromatin to regulate expression of specific target genes. Previous reports have demonstrated an interaction between PcG proteins and members of the general transcriptional machinery, suggesting that binding of PcG complexes to promoters may be stabilized by these interactions (Breiling et al. 2001; Saurin et al. 2001). However, it is likely that a HKMT-containing PRC complex must first be recruited by a sequence-specific DNA-binding protein.
Because none of the HKMT-containing PRC complexes contain a site-specific DNA-binding protein, target genes cannot be identified using a bioinformatics approach to search for consensus binding sites in the genome. Therefore, we have used two global screening methods to identify genes that are regulated by mammalian PRC2/3 complexes. First, using oligonucleotide cDNA arrays, we identified genes whose expression changes upon siRNA-mediated depletion of Suz12. Second, we used a combination of chromatin immunoprecipitation and genomic microarrays to identify promoters bound by Suz12. Interestingly, we show that multiple components of the PRC2/3 complexes colocalize on these newly identified target genes and that binding of these proteins to target genes correlates with methylation of $\mathrm{H} 3-\mathrm{K} 27$.

\section{Results}

Suz12 protein levels are increased in human colon tumors

Although Drosophila PcG proteins have been mostly implicated in transcriptional control during development, human PcG proteins have been linked to cancer development. For example, previous studies have demonstrated that Ezh2 is overexpressed in various human cancers (Varambally et al. 2002; Bracken et al. 2003; Kleer et al. 2003). Additionally, in endometrial stromal tumors, a fragment of the Suz12 gene is frequently fused to a second gene encoding a zinc finger protein (Koontz et al. 2001). In our previous studies, we have observed up-regulation of Suz12 mRNA in tumors of the colon, breast, and liver (Kirmizis et al. 2003). Specifically, we found that eight of nine colon cancer patients tested had high levels of Suz12 mRNA in the tumor tissue as compared with the normal tissue. Furthermore, we showed that the up-regulation of Suz12 mRNA is mediated by the $\mathrm{TCF} / \beta$-catenin transcription complex (Kirmizis et al. 2003), whose increased activity is responsible for the development of the majority of human colon cancers (for review, see Bienz and Clevers 2000). The fact that Suz12 mRNA is present at high levels in colon tumors suggests that colon cancer provides a good model system to identify PRC target genes. However, it was first necessary to determine if the increased Suz12 mRNA expression in colon tumors correlates with increased Suz12 protein levels. We developed an antibody against the Suz12 protein and demonstrated that it recognizes the correct protein, using cells transfected with a plasmid expressing an HA-tagged Suz12. Western analysis showed that the HA antibody recognized a 93-kDa protein in cells transfected with HA-Suz12 but not in cells transfected with the empty vector (Fig. 1A, left panel). The purified Suz12 antibody recognized the same protein in both transfected cell populations (Fig. 1A, right panel). This result is expected because the endogenous protein runs the same size as the HA-tagged protein. We next analyzed wholetissue extracts from five different colon cancer patients for Suz12 protein levels. As shown in Figure 1B, four out of five samples showed increased levels of Suz12 in the 
Figure 1. Suz12 protein expression is elevated in human colon tumors. $(A)$ Western blot analysis to demonstrate the specificity of the Suz12 antibody. SW480 cells were transfected with an HA-Suz12 or a control (HA-empty) construct and subjected to immunoblot analysis. Two separate blots were prepared and probed with either an HA antibody (left panel) or a Suz12 antibody (right panel). The HA antibody recognizes only the HA-Suz12 protein and the Suz12 antibody detects both the HA-Suz12 and endogenous Suz12 proteins. (B) Western blot analysis of whole-tissue extracts prepared from normal $(\mathrm{N})$ and tumor $(\mathrm{T})$ tissues of five different colon cancer patients. The blot was first probed with an anti-Suz12 antibody and then reprobed with an antiactin antibody as a loading control. The arrowhead points to the band with the correct size of Suz12 193 $\mathrm{kDa}$. A second lower band, detected with the Suz12 antibody in patients $1-3$, probably represents a protein degradation product.

tumor tissue as compared with adjacent normal tissue. One patient had high Suz12 levels in both the normal and tumor tissues, and, therefore, it is possible that the normal sample was contaminated with tumor tissue. These findings are in agreement with our previous results showing up-regulation of Suz12 mRNA in colon tumors. In three of our extract preparations, the antibody recognized the $93-\mathrm{kDa}$ band plus a lower band of $\sim 85$ $\mathrm{kDa}$. This lower band has also been detected by others using a different Suz12 antibody (Tie et al. 2003). It is possible that the lower band represents a Suz12 degradation product that is produced during extraction of the tumor tissue because we have not detected it in nuclear extracts prepared from cell lines (data not shown).

\section{Depletion of Suz12 in colon cancer cells results in alteration of gene expression}

Recent studies have shown that Suz12 is a component of the PRC2/3 complexes (Cao et al. 2002; Czermin et al. 2002; Kuzmichev et al. 2002, 2004; Muller et al. 2002), suggesting that up-regulation of Suz12 in colon cancers may modulate the tumor phenotype by modifying histones and thus changing gene expression profiles. To examine this hypothesis, we used RNA interference (RNAi) to decrease Suz12 expression in colon cancer cells. We designed small interfering RNAs (siRNAs) to target the Suz12 transcript and tested them using two human colon cancer cell lines (Fig. 2; data not shown). We observed a decrease in both Suz12 mRNA and protein levels by $24 \mathrm{~h}$ after transfecting cells with the Suz12 siRNAs (Fig. 2A,B, cf. lanes 1 and 4). The down-regulation of Suz12 was more prominent after $72 \mathrm{~h}$ of transfection (Fig. 2A,B, cf. lanes 3 and 6). Lamin A/C and green fluorescent protein (GFP) siRNA duplexes did not affect the amounts of Suz12 verifying the specificity of the RNAi approach. We next examined the phenotype of the colon cancer cells having reduced Suz12 expression. We did not detect any changes in the morphology or the
A
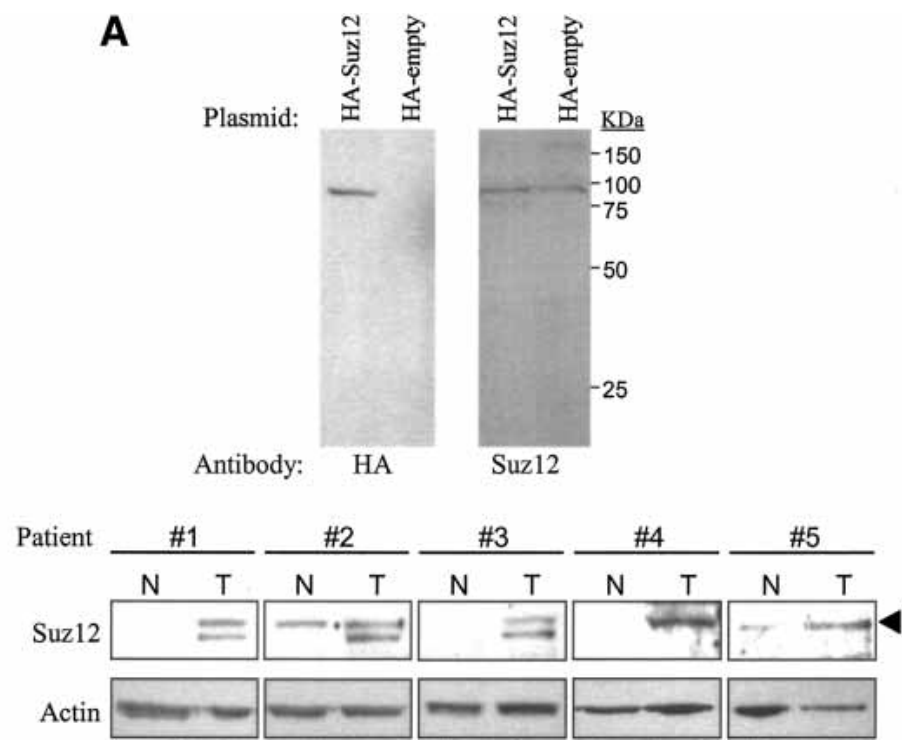

cell cycle profile of the cells after treatment with Suz12 siRNAs for $72 \mathrm{~h}$ (data not shown). However, RNAi does not eliminate Suz12 expression entirely from the cells, and the remaining Suz12 may be sufficient to mask any obvious phenotypes, especially in a short-term assay. It is also possible that certain functions of Suz12 in these cells are redundant with another protein, and, therefore, removing only Suz12 is not enough to cause changes in cell proliferation.

To determine whether short-term removal of Suz12 from cells results in altered transcription profiles, we

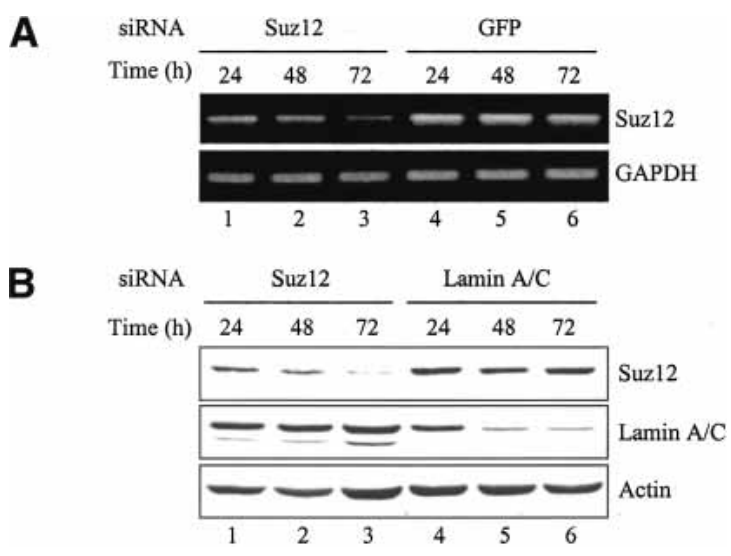

Figure 2. Suz12 expression in colon cancer cells is disrupted by RNA interference. (A) RT-PCR analysis on total RNA extracted from SW480 cells at various time points after transfection with Suz12 or GFP siRNA duplexes. Primers specific to the GAPDH mRNA were used in RT-PCR to ensure that the RNA was correctly quantitated. (B) Western blot analysis on whole-cell extracts prepared from SW480 cells at various time points after transfection with Suz12 or Lamin A/C siRNA duplexes. Antibodies against Suz12 and Lamin A/C were used to show specific depletion of the respective proteins. The blot was also probed with the actin antibody to demonstrate equal loading of protein samples. 
performed RNAi experiments and monitored global gene expression using oligonucleotide microarrays. We transfected SW480 colon cancer cells with either Suz12 or GFP siRNAs and harvested total RNA after $48 \mathrm{~h}$. Although depletion of Suz12 was best at $72 \mathrm{~h}$ (Fig. 2), we chose to monitor expression at the 48 -h time point to minimize indirect effects of the Suz12 down-regulation. Three independent experiments were performed with the Suz12 siRNAs or the GFP siRNA, and the three RNA preparations from each treatment were pooled into one sample to reduce the experimental variation. The pooled Suz12-siRNA and GFP-siRNA samples were then labeled with a fluorescent dye and hybridized onto four separate Affymetrix U133A gene chips (Fig. 3A). Using the default settings on the microarray analysis software, we selected the genes whose expression was significantly altered by loss of Suz12. We found 46 up-regulated transcripts (representing 35 unique genes) and 23 downregulated transcripts (representing 15 unique genes) in cells having reduced Suz12 levels (Fig. 3B). A list of the
UniGene IDs and fold changes of the 46 up-regulated and 23 down-regulated mRNAs is provided as Supplemental Material (Supplementary Table S1). Two of the transcripts that were down-regulated correspond to Suz12 and thus verify the RNAi results obtained using RTPCR (Fig. 2B). To confirm the results obtained in the microarray analyses, we selected 14 genes that represented the entire range of the fold changes (both up and down) observed. RNA was prepared from cells treated with siRNAs either to Suz12 or to GFP, using samples independent from those used for the microarray analyses. RT-PCR analysis demonstrated that the genes identified on the arrays show the expected response to the Suz12 siRNA treatment in the independent experiment (Fig. 3C).

\section{Suz12 binds directly to promoters}

The experiments described above show that down-regulation of Suz12 alters the expression level of multiple
A

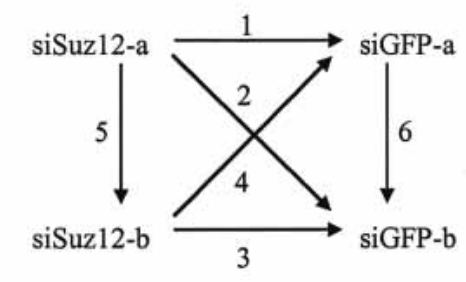

C
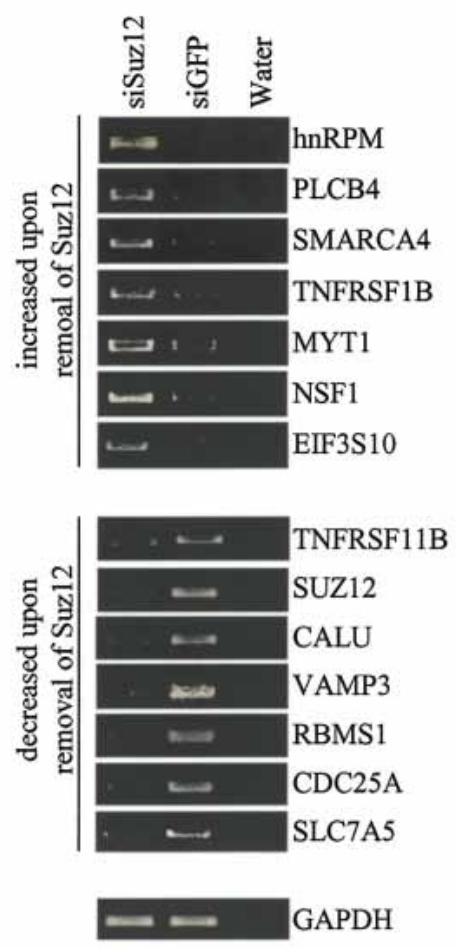

B

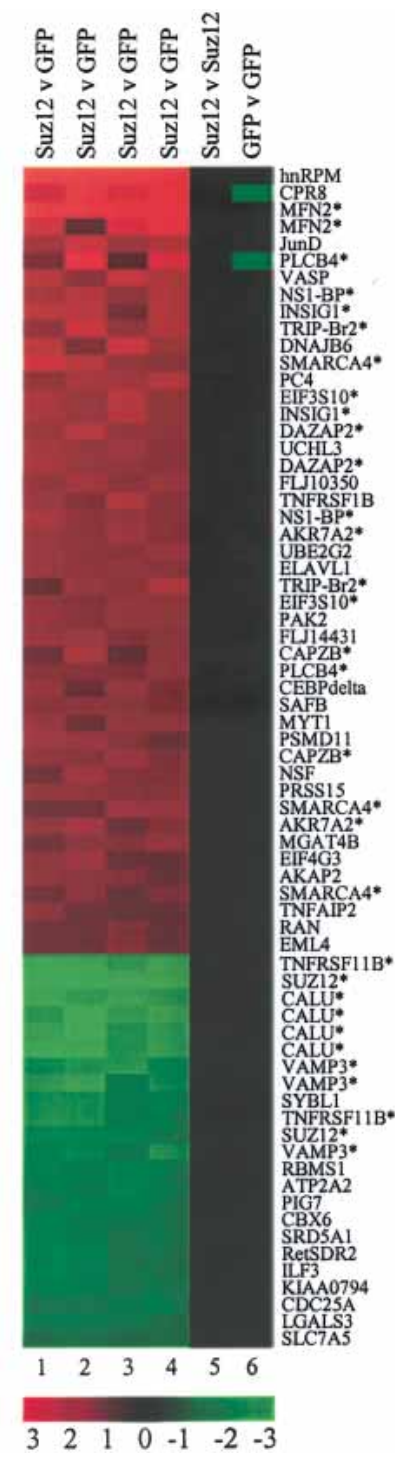

Figure 3. Removal of Suz12 from SW480 cells results in alteration of gene expression. (A) Schematic showing the comparisons among the four Affymetrix U133A arrays that were used to analyze gene expression in cells incubated with either Suz12 or GFP siRNAs. The numbers on the arrows correspond to the columns in B. Columns 1, 2, 3, and 4 represent siSuz12 versus siGFP comparisons and thus allow identification of up- and downregulated genes. Columns 5 and 6 are comparisons of identical samples; these should show no variation and were used to identify false positives. $(B)$ Tree-view diagram depicting the genes that were significantly deregulated upon Suz12 depletion. Red represents up-regulated genes, and green represents down-regulated genes in the Suz12 RNAi samples in reference to the GFP RNAi samples. The numbers on the color scheme represent the fold changes in gene expression. Genes denoted with an asterisk $\left({ }^{\star}\right)$ were identified more than once on the microarrays. $(C)$ RT-PCR analysis of independent RNAi experiments confirming the results obtained using the microarrays. Primers specific to GAPDH mRNA were used in RTPCR to ensure that the RNA was correctly quantitated. 
genes. However, these findings do not demonstrate if Suz12 controls the expression of these genes directly by binding to their promoters. It is possible that removal of Suz12 from the cells changes the expression of these genes through an indirect mechanism. Therefore, we used chromatin immunoprecipitation (ChIP) to investigate whether Suz12 is recruited to the promoters of the deregulated genes. Because Suz12 is a component of transcriptional repressive complexes, we focused our initial investigation on the genes whose expression increased upon depletion of Suz12 from the cells. We performed ChIP experiments in SW480 cells using antibodies to Suz12, RNA polymerase II (Pol II), and a nonspecific IgG control. Using PCR primers that spanned the transcriptional start site, we monitored binding of Suz12 and Pol II to six promoters that drive expression of mRNAs that were up-regulated upon loss of Suz12. Two of the genes, Myelin transcription factor 1 (MYT1) and N-ethylmaleimide-sensitive factor (NSF), are expressed from two different transcriptional start sites separated by several kilobases. Therefore, we monitored binding of Suz12 to both the proximal and distal promoter regions of these two genes. Suz12 showed robust binding to the distal promoter region of MYT1 but not to the proximal promoter region (Fig. 4, lane 3). We did not detect strong binding of Suz12 to the distal or the proximal promoter region of the NSF gene or at the other four tested promoters. Studying specific protein/DNA interactions through ChIP assays requires the use of a highly specific antibody. The specificity of the Suz12 antibody was first

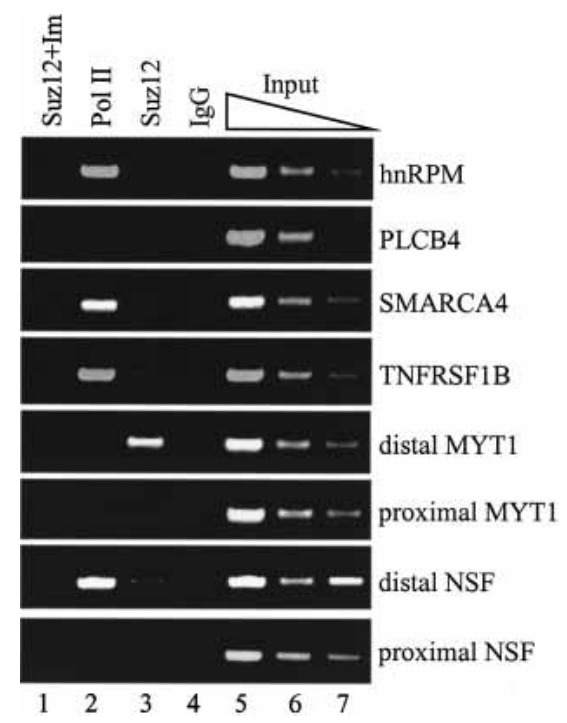

Figure 4. The MYT1 promoter is directly regulated by Suz12. Chromatin immunoprecipitation experiments were performed in SW480 cells using antibodies to RNA Polymerase II (lane 2), Suz12 (lane 3), and a control IgG (lane 4). A control immunoprecipitation was also performed with a Suz12 antibody that was preincubated with the Suz12 immunogen at a 10 -fold excess by weight (lane 1). Three dilutions of the total input are also shown (lanes 5-7). The precipitated chromatin was analyzed with PCR using primers specific to the promoters of the indicated genes. illustrated by the fact that it recognized a single protein band by immunoblot analysis in nuclear extract preparations of cultured cells (data not shown) and by the fact that the protein detected on the Western blot is diminished after introduction of Suz12 siRNAs to the cells (Fig. 2B). To further demonstrate the specificity of the Suz12 antibody, we performed a ChIP assay in which the antibody was preincubated with the Suz12 immunogen (a fragment of Suz12 protein used to develop the antibodyl at a 10-fold excess by weight. The immunogen abrogated the ability of the Suz12 antibody to enrich the MYT1 distal promoter (Fig. 4, lane 1). Collectively, these results demonstrate that the Suz12 antibody used in the ChIP experiments specifically enriches selected chromatin fragments. Therefore, the ChIP analysis demonstrated that MYT1 expression is directly regulated by Suz12, whereas expression of the other five tested genes may be indirectly regulated by Suz12.

The above ChIP experiments allowed the identification of MYT1 as the first direct target gene of Suz12. However, similar analysis for detecting Suz12 binding to the remaining genes identified in the gene expression microarrays would require preparation of numerous PCR primers. Additionally, the lack of any known cis element responsible for recruiting Suz12 to chromatin does not allow us, by sequence examination, to determine where to place the primers for optimal detection of Suz12 on these loci. To circumvent these caveats, we combined the ChIP analysis with custom oligonucleotide promoter microarrays. The custom arrays contained probes spaced at a high density that represented 5 -kb promoter regions (4 kb upstream to $1 \mathrm{~kb}$ downstream relative to the transcriptional start site) of all the up-regulated and downregulated genes that were identified in the gene expression study described above. This combination of ChIP with custom promoter arrays will identify the genes that are directly regulated by Suz12 and precisely localize the binding of Suz12 to these promoters in a high-throughput manner. We first performed a standard ChIP assay in SW480 cells using the Suz12 antibody and a nonspecific IgG as a control. The immunoprecipitated chromatin was examined using MYT1 promoter primers to ensure specific enrichment of Suz12-bound DNA fragments (data not shown). We next prepared amplicons of $\sim 500 \mathrm{bp}$ from the Suz12- and IgG-immunoprecipitated chromatin samples as well as from an input chromatin reference sample (see Fig. 7C, right panel [below]). The amplicons were labeled with a fluorescent dye and hybridized onto separate custom promoter arrays. To identify the genes that are directly regulated by Suz12, we compared the hybridization intensities between the Suz12 array and the input array and calculated a Suz12/Input ratio for each oligonucleotide probe. Regions with a Suz12/Input ratio higher than 2 were considered positive for Suz12 binding. To eliminate from consideration promoter regions that are enriched nonspecifically during the ChIP procedure, we also calculated an $\mathrm{IgG} /$ Input ratio for each oligonucleotide probe. We performed three independent analyses and found eight promoters that were repeatedly enriched by the Suz12 antibody suggesting that those 
genes are directly regulated by Suz12 (Table 1). Profiles from a representative experiment of four of the eight positive promoters are shown in Figure 5. Use of the high-density oligonucleotide arrays allowed for more precise localization of Suz12 recruitment to its target loci than did the standard ChIP experiments. The small size of the amplicons in combination with the high density of the custom oligonucleotide arrays mapped the binding site of Suz12 within 500-bp regions. The results from these ChIP-chip experiments were validated in independent ChIP experiments using PCR primers that spanned the regions showing the highest enrichment by the Suz12 antibody (Fig. 5, inserts). As expected, analysis of the MYT1 promoter showed specific recruitment of Suz12. Interestingly, Suz12 binding to MYT1, as well as to some of the other promoters, occurred at two distinct promoter regions; one near the transcriptional start site and one farther upstream (e.g., MYT1 and SYBL1). The two occupied regions on these promoters were separated by several hundred bases, suggesting that Suz12 may associate with the transcriptional start site via a DNA looping mechanism mediated by protein-protein interactions that is initiated by specific binding of Suz12 to an upstream region. Such a looping mechanism has been proposed to mediate the interaction of Drosophila PcG proteins with their target promoters (Orlando et al. 1998). However, it remains possible that Suz12 is recruited specifically to the region near the start site via a site-specific DNA-binding protein. Interestingly, we observed direct recruitment of Suz12 to genes that were

Table 1. Confirmed Suz12 target genes

\begin{tabular}{|c|c|c|c|}
\hline No. & \multicolumn{2}{|l|}{ Gene name } & Position(s) of enrichment \\
\hline \multicolumn{4}{|c|}{ Targets identified by oligonucleotide expression arrays } \\
\hline \multicolumn{4}{|c|}{ Genes repressed by SUZ12 } \\
\hline 1 & EIF3S10 & Eukaryotic translation initiation factor 3 & -500 \\
\hline 2 & MYT1 & Myelin transcription factor & -1700 and +1 \\
\hline 3 & PSMD11 & Proteasome 26S subunit non-ATPase 11 & -1000 and +500 \\
\hline 4 & MGAT4B & Mannosyl glycoprotein acetylglucosaminyltransferase 4B & -3500 and -1000 \\
\hline 5 & EML4 & Echinoderm microtubule-associated-protein-like 4 & -500 \\
\hline \multicolumn{4}{|c|}{ Genes activated by SUZ12 } \\
\hline 6 & SYBL1 & Synaptobrevin-like 1 & -2000 and +1 \\
\hline 7 & SRD6A1 & Steroid-5- $\alpha$-reductase, $\alpha$ polypeptide 1 & +1 \\
\hline 8 & RBMS1 & RNA-binding motif single-stranded interacting protein 1 & -2000 \\
\hline \multicolumn{4}{|c|}{ Targets identified by CpG island arrays } \\
\hline 1 & DMRTA2 & Doublesex-mab-3 (DM) domain-like family A2 & -4000 and -2000 \\
\hline 2 & KCNA1 & Potassium voltage-gated channel & -2000 \\
\hline 3 & LOC119392 & Hypothetical protein & +1 \\
\hline 4 & MFAP1 & Microfibrillar-associated protein & +1 \\
\hline 5 & LOC115811 & Similar to RIKEN cDNA 4933433C09 & +200 \\
\hline 6 & CNTFR & Ciliary neurotrophic factor receptor & $-2000,-1000$, and +1 \\
\hline 7 & KIAA0254 & Sorting nexin 19 & -1000 \\
\hline 8 & TRIM52 & Tripartite motif-containing 52 & +1 \\
\hline 9 & $\mathrm{RDC} 1$ & G-protein-coupled receptor & +1 \\
\hline 10 & WNT1 & Wingless-type MMTV integration site member 1 & -2000 and +500 \\
\hline 11 & KIF2C & Kinesin family member $2 \mathrm{C}$ & +1 \\
\hline 12 & HSPC228 & Chromosome 6 open reading frame 55 & +1 \\
\hline 13 & KIS & Kinase interacting with stathmin & +1 \\
\hline 14 & SLC25A3 & Solute carrier family 25 member 3 & +300 \\
\hline 15 & $\mathrm{NKX} 2 \mathrm{C}$ & NK2 transcription factor related lcous 3 & +600 \\
\hline 16 & AK056349 & Cholinergic receptor, muscarinic 3 & -200 \\
\hline 17 & SRP9 & Signal recognition particle $9 \mathrm{KDa}$ & +1 \\
\hline 18 & NUDT2 & Nudix-type motif 2 & -3000 and +1 \\
\hline 19 & $\mathrm{CNR} 1^{\star}$ & Cannabinoid receptor 1 & +1 \\
\hline 20 & RBMS1 3'-end & RNA-binding motif single-strand interacting protein & +95000 \\
\hline
\end{tabular}

The position of enrichment indicates the location of Suz12 binding in the promoter relative to the transcriptional start site, which is represented by +1 . The top part indicates the genes that were identified by the gene expression analysis (Fig. 3), and the bottom part indicates the genes that were identified by the ChIP-CpG-island arrays (Fig. 6). For the genes identified using the gene expression analyses (Fig. 3), the region spanning from $-4 \mathrm{~kb}$ to $+1 \mathrm{~kb}$ relative to the start site was represented on the custom oligonucleotide arrays. Most of the CpG islands detected by the ChIP-CpG analyses (Fig. 6) corresponded to promoter regions. For these cases, $-4 \mathrm{~kb}$ to $+1 \mathrm{~kb}$ relative to the start site was analyzed on the custom arrays. However, in some cases the CpG island was located $3^{\prime}$ of a gene (e.g., $R B M S 1)$. In these cases, a $5-\mathrm{kb}$ region surrounding the $\mathrm{CpG}$ clone was represented on the arrays. The CNR promoter, indicated with an asterisk $\left({ }^{\star}\right)$, was not included in the custom oligonucleotide arrays, but Suz12 binding to that promoter was confirmed by PCR analysis. 
Figure 5. Mapping of Suz12 binding to its target promoters. Oligonucleotide microarrays containing probes that represent a $5-\mathrm{kb}$ region from each promoter of the identified Suz12regulated genes (Fig. 3B) were prepared. The arrays were hybridized with amplicons prepared from ChIP experiments using a Suz12 antibody, a nonspecific IgG antibody, and an input control. Shown are six promoter regions: three that were up-regulated (MYT1, EIF3S10, PLCB4) upon removal of Suz12 from cells and three that were down-regulated (SYBL1, RBMS1, RetSDR) upon removal of Suz12 from cells. The fold enrichment was calculated by dividing the Suz12 or IgG hybridization intensity signal by the input control signal for each oligonucleotide probe. The inserts within each graph show independent ChIP confirmation using PCR analysis. The primers used in the PCR analysis were designed to span the region showing the highest peak of enrichment for each promoter. A complete list of the Suz12bound promoters and the position of binding is shown in Table 1.
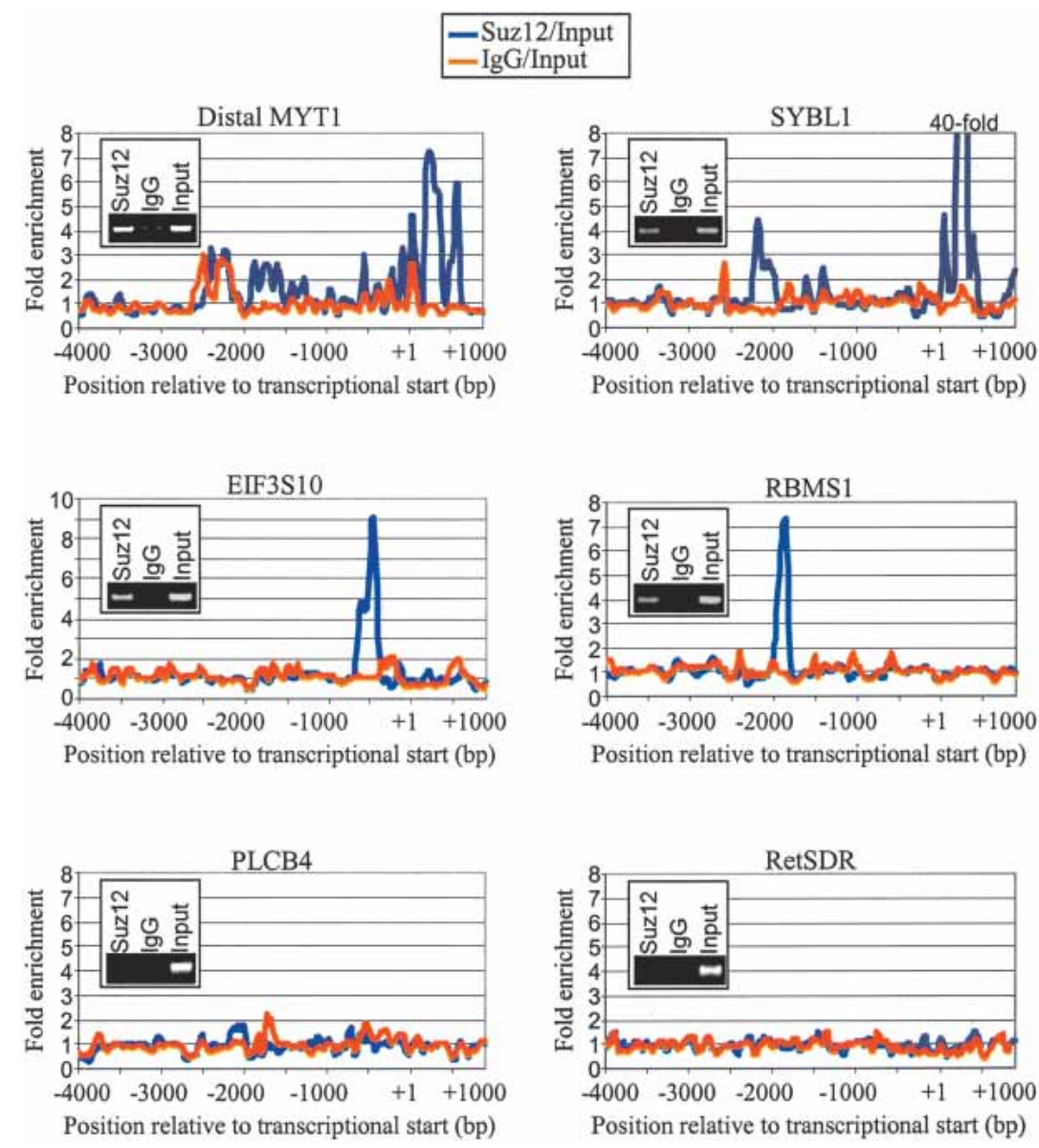
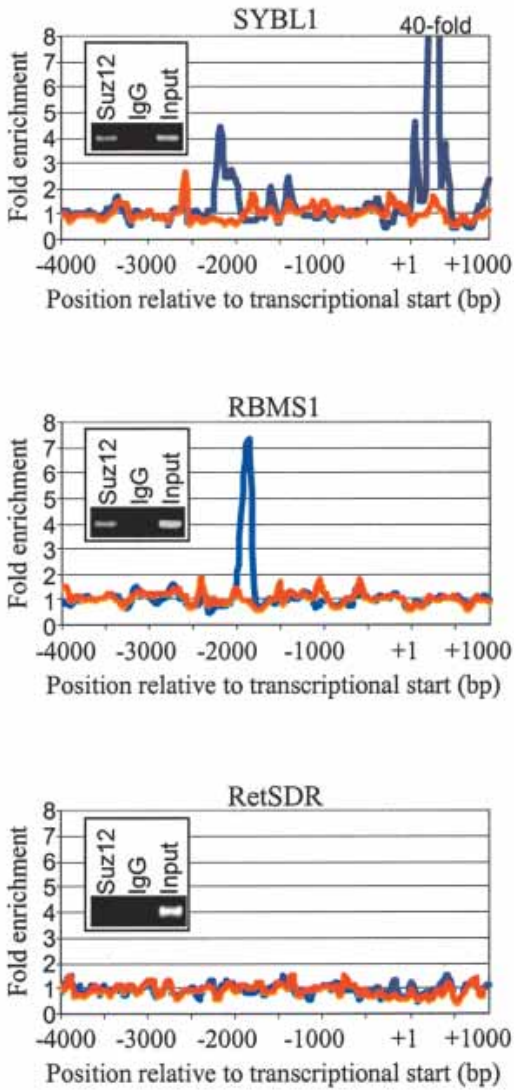

up-regulated (MYT1 and EIF3S10) as well as down-regulated (SYBL1 and RBMS1) after depletion of Suz12 from SW480 cells, indicating that Suz12 might have a role in both transcriptional repression and activation. The majority of the genes tested did not show Suz12 binding (e.g., PLCB4 and RetSDR), suggesting that expression of those genes is indirectly regulated by Suz12. However, it remains possible that Suz12 binds to a region farther upstream than $-4 \mathrm{~kb}$ or farther downstream than $+1 \mathrm{~kb}$ in these promoters.

\section{Identification of additional Suz12 target promoters using CpG-island microarrays}

Although the experiments described above identified a small set of direct target genes of Suz12, they did not identify numerous high-affinity binding sites. As noted above, this could be caused by the fact that most of the genes identified in the siRNA experiments are indirectly regulated by Suz12. Therefore, to identify additional Suz12 targets, we used a ChIP-CpG-island microarray technique that has previously been used in our laboratory to identify genomic loci bound by E2F family members (Weinmann et al. 2002; Oberley et al. 2003; Wells et al. 2003). This technique will identify direct Suz12 targets that are within several kilobases of the CpG islands present on the microarray. Using the Suz12 antibody and preimmune (PI) serum as a control, we performed a standard ChIP assay in SW480 cells. We next prepared amplicons from the Suz12- and PI-immunoprecipitated chromatin samples as well as from an input chromatin reference sample. The amplicons from the immunoprecipitated samples were labeled with one fluorescent dye, whereas the reference sample (input) was labeled with a different fluorescent dye. To identify Suz12-bound loci, we applied the Suz12 and input amplicons to an array containing $\sim 12,000 \mathrm{CpG}$ islands. We selected all the CpG clones that had intensity signals from the Suz12 chromatin at least threefold higher than the input control (Fig. 6A). To control for CpG clones that were enriched nonspecifically by the ChIP procedure, we hybridized a second array with the preimmune versus input amplicons. CpG islands that were enriched by the preimmune serum were discarded from further analysis. This procedure was repeated twice, and 52 CpG clones were selected as positives. Next, we used the University of California-Santa Cruz Genome Browser (http:// genome.ucsc.edu) to determine the genomic location of all the CpG clones. Out of 52 clones, 41 contained sequences that matched to locations near promoters or $3^{\prime}$ ends of known and predicted genes (Supplementary Table S2). Because there is some degree of redundancy of the $\mathrm{CpG}$ islands printed on the arrays, three gene pro- 


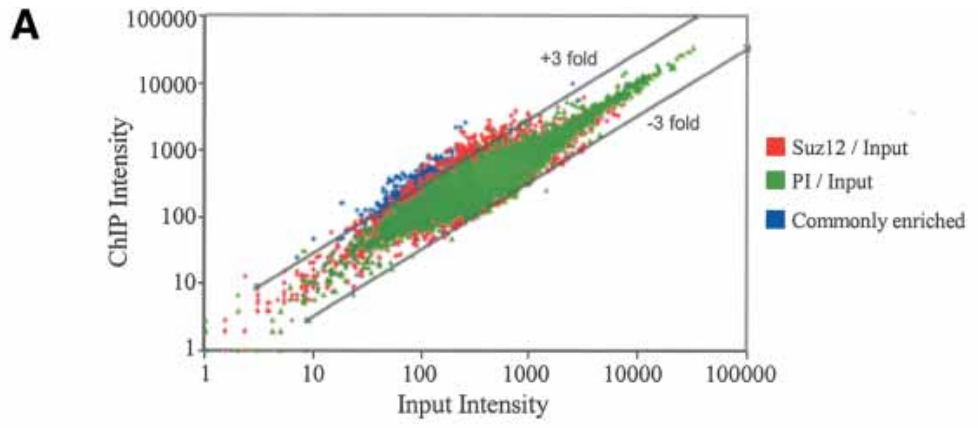

B
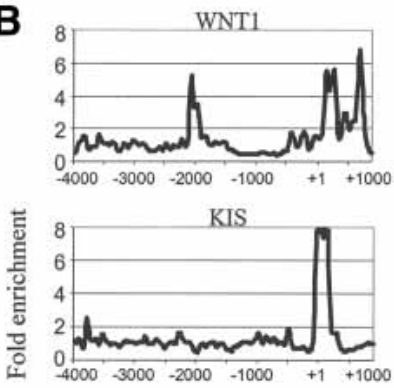

TLK2

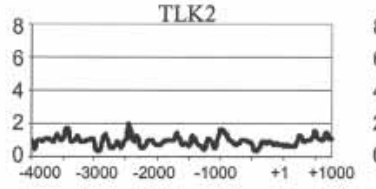

Position relative to transcriptional start (bp)

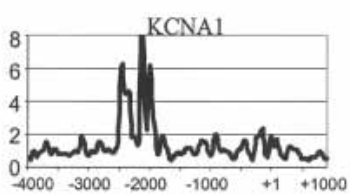

KIF2C

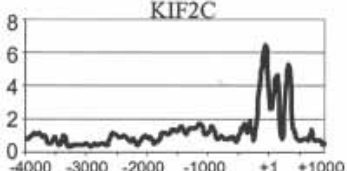

PVALB

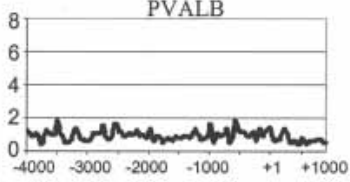$$
\text { p) }
$$

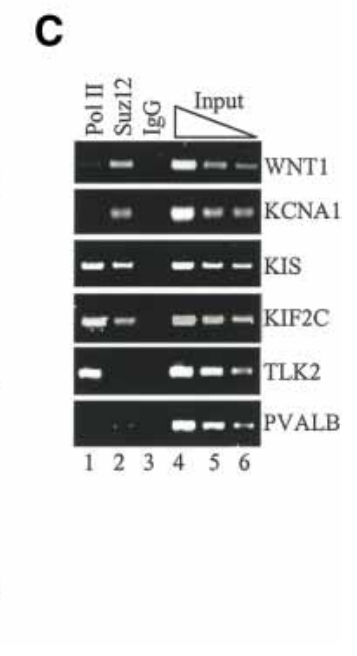

Figure 6. Identification of additional direct Suz12 target genes. (A) Scatter plot showing the intensities of the spots on the CpG-island microarrays. Two independent experiments were performed to identify Suz12bound loci in SW480 cells. In red are the spot intensities from a representative array that was hybridized with Suz12 and input amplicons. Shown in green are the spot intensities of a representative array that was hybridized with IgG and input amplicons. Red spots that enriched at least threefold were selected for further analysis. The spots shown in blue were enriched at least threefold in both the Suz12 and IgG control immunoprecipitations and represent DNA fragments that precipitate nonspecifically in the ChIP procedure. $(B)$ Confirmation and mapping of Suz12 binding to a $5-\mathrm{kb}$ region of each promoter identified in $A$ was performed using custom oligonucleotide promoter arrays. The arrays were prepared and hybridized as in Figure 5. Shown are the fold enrichments from the Suz12 and input comparisons of six promoters: four true positive targets and two (TLK2 and PVALB) false positives. $(C)$ Confirmation of the results shown in $B$ using PCR analysis. Independent ChIP experiments were performed in SW480 cells using antibodies to RNA Polymerase II (lane 1), Suz12 (lane 2), and a control IgG (lane 3). Three dilutions of the input chromatin are also shown (lanes 4-6). The precipitated chromatin was analyzed with PCR using primers specific to the promoters of the indicated genes. moters were identified twice as unique clones. Also, three clones contained sequences that matched to two different gene promoters. Probably during the construction of the CpG-island library, DNA fragments representing different $\mathrm{CpG}$ islands were introduced in tandem within one clone. Additionally, two CpG clones did not match to any genomic loci, three matched to sequences that were not near annotated genes, and six matched to repeat elements; these 11 clones are not listed in Supplementary Table S2.

To determine if the identified CpG islands are real targets of Suz12 and to precisely map the binding of Suz12 to the CpG islands, we again coupled the ChIP assay with custom oligonucleotide promoter arrays. These custom arrays represented $5-\mathrm{kb}$ regions of the promoters of all the genes that were associated with the identified $\mathrm{CpG}$ islands. Using the same triplicate preparations of Suz12, IgG, and Input amplicons used in the previous experiments, we hybridized the custom promoter arrays; the results are summarized in Table 1. Of the 41 promoters represented on the arrays, 20 showed robust binding of Suz12 (Table 1). As shown in Figure 6B, some promoters showed enrichment at multiple loci (such as WNT1), whereas others were bound by Suz12 only at one locus (e.g., KCNA1, KIS, and KIF2C). The remaining 21 promoters that did not bind to Suz12, such as TLK2 and PVALB, either represent false-positive clones selected in the CpG-island array experiments or are bound by Suz12 outside the 5 -kb region that was printed on the custom promoter arrays (e.g., at $+2 \mathrm{~kb}$ ). The results obtained using the custom promoter arrays were further validated in independent ChIP experiments using PCR analysis (Fig. 6C). ChIP analysis demonstrates that two of the promoters show robust binding of Suz12 and almost no binding of Pol II, a pattern similar to that seen on the MYT1 distal promoter. Interestingly, two other promoters show moderate binding of Suz12 and robust binding of Pol II. Finally, two of the genes show no binding of Suz12.

\section{PRC-mediated repression of Suz12 target genes is associated with methylation of H3-K27}

Recent studies have shown that Suz12 is a component of two different PRC complexes, each of which contains Ezh2, an HKMT that is associated with transcriptional silencing, and at least one form of Eed (Kuzmichev et al. 2004). Therefore, we examined binding of Suz12, Ezh2, and Eed along a 5-kb region around the distal MYT1 promoter (Fig. 7A). For these experiments, we generated am- 
A

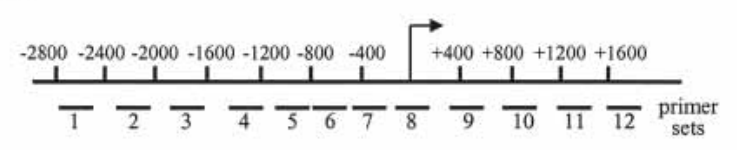

B
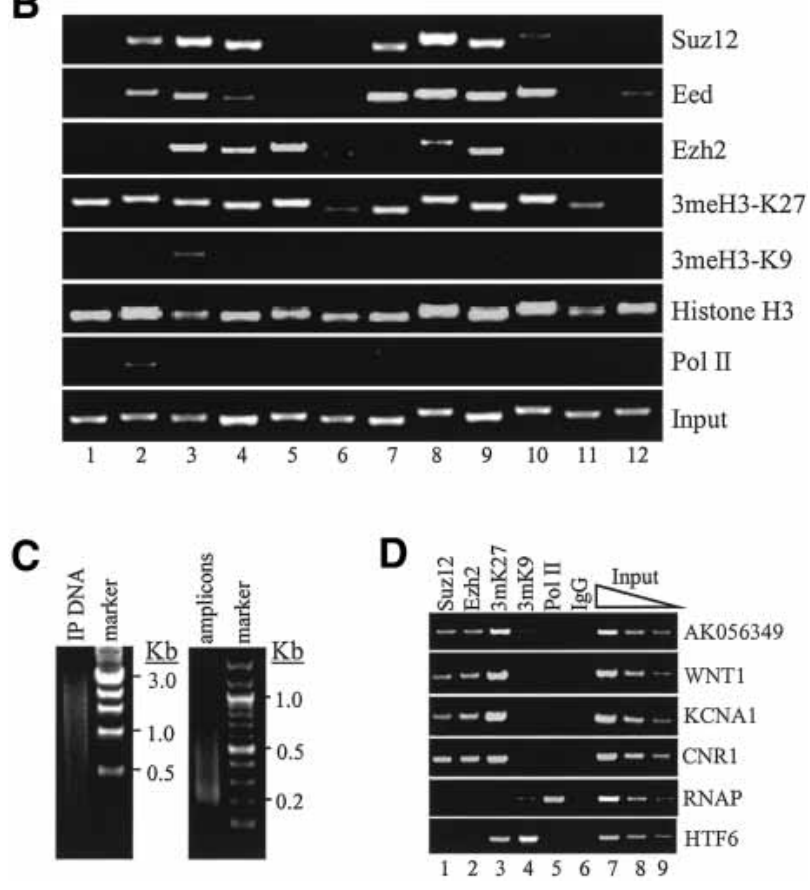

Figure 7. The Suz12 target promoters are bound by other PRC2/3 components and are methylated at Lys 27 of histone H3. (A) A schematic of the distal MYT1 promoter locus is shown; the positive and negative numbers indicate the position in the promoter relative to the transcriptional start site represented by the arrow. The lines below the MYT1 locus represent the promoter fragments amplified by the different PCR primer pairs. (B) ChIP experiments were performed in SW480 cells using antibodies to Suz12, Eed, Ezh2, tri-methyl H3-K27, trimethyl H3-K9, histone H3, and RNA Polymerase II. The immunoprecipitated chromatin from each ChIP sample was used to prepare amplicons by LM-PCR as described in Materials and Methods. The amplicons were then analyzed by PCR. The number of each lane corresponds to the primer pair indicated in $A$. Amplicons prepared from input chromatin were used as a positive control. (C) Shown are examples of the size of DNA from a ChIP experiment (left panel) and amplicons prepared from a typical LM-PCR reaction (right panel). (D) ChIP experiments in SW480 cells using the same antibodies as in B. A nonspecific IgG antibody (lane 6) was used as a negative control, and three dilutions of the input chromatin (lanes 7-9) were used as positive controls to demonstrate linear amplification. The immunoprecipitated chromatin was analyzed by PCR using primers specific to the promoters of the genes indicated.

plicons from ChIP samples by LM-PCR and used them in conventional PCR using promoter-specific primers. This type of analysis allows precise localization of the binding of a factor to a promoter region because, as shown in Figure 7C, amplicons have a smaller range in size (200-500 bp) as compared with immunoprecipitated
DNA fragments $(1-3 \mathrm{~kb})$ isolated from a standard ChIP assay. Using this analysis, we showed that Suz12 binds to two regions in the 5-kb sequence; one near the distal transcriptional start site and one several kilobases upstream (Fig. 7B). These results are in complete agreement with our findings from the experiments using the custom oligonucleotide arrays (cf. Figs. 7B and 5). Using this high-resolution PCR analysis, we also found that Ezh2 and Eed colocalize with Suz12 on the MYT1 promoter, confirming that MYT1 is a target gene of the PRC complexes (Fig. 7B). To determine if the recruitment of the PRC complexes correlates with the HKMT activity of Ezh2, we next examined the methylation of H3-K27 at the $5-\mathrm{kb}$ region of the MYT1 promoter. We show that binding of Suz12, Ezh2, and Eed to the MYT1 promoter correlates with, but does not precisely overlap, trimethylation of H3-K27. This result suggests that the PRC complexes can methylate histones located at the PRCbinding sites and in the surrounding region. Interestingly, no methylation of H3-K9 was detected at the repressed MYT1 locus, indicating that in vivo PRC-mediated silencing is associated with trimethylation of Lys 27 but not Lys 9 of histone H3 (Fig. 7B), as previously suggested (Kuzmichev et al. 2002). The results of the siRNA experiments (demonstrating increased MYT1 mRNA in the absence of Suz12) and ChIP experiments (demonstrating methylation of $\mathrm{H} 3-\mathrm{K} 27$ ) suggest that the MYT1 promoter is kept in a repressed state by its association with the PRC2/3 complexes. Interestingly, we could not detect binding of Pol II to any location within the $5-\mathrm{kb}$ sequence surrounding the MYT1 distal promoter. This lack of Pol II suggests a model for the PRC-mediated regulation of the MYT1 promoter; first, recruitment of PRC2/3 causes methylation of $\mathrm{H} 3-\mathrm{K} 27$, which then results in the formation of a chromatin structure that is incompatible with binding of Pol II, and thus the formation of a transcription initiation complex is prevented.

The robust Suz12 and weak Pol II signals seen on four of the promoters identified on the CpG-island arrays (Fig. $6 \mathrm{C}$; data not shown) suggest that they may be regulated by a PRC complex in a manner similar to that of the MYT1 promoter. To test this hypothesis, we examined binding of Ezh2 to the AK056349, Wnt1, KCNA1, and CNR1 promoters. As shown in Figure 7D, Ezh2 is recruited to all of these Suz12 target promoters. Interestingly, binding of Suz12 and Ezh2 correlates with methylation of $\mathrm{H} 3-\mathrm{K} 27$, but not of $\mathrm{H} 3-\mathrm{K} 9$, at these four promoters. To demonstrate the specificity of our antibodies, we analyzed the methylation status of two other promoters, one active (the largest subunit of Pol $I I$ ) and one inactive (the pericentromeric gene HTF6), in SW480 cells. As expected, the Pol II promoter was bound by Pol II but not by Suz12 or Ezh2 and showed no methylation of $\mathrm{H} 3-\mathrm{K} 27$ or H3-K9. In contrast, the silenced pericentromeric HTF6 promoter was characterized by methylation of H3-K27 and H3-K9. Methylation of H3-K27 at the HTF6 promoter may be mediated by a methyltransferase activity other than that of the PRC complexes because Suz12 and Ezh2 were not bound to the promoter (Fig. 7D). Alternatively, the kinetics of binding of an 
Ezh2-containing complex to this promoter may be different from the other promoters studied.

Our RNAi experiments described above showed that removal of Suz12 from colon cancer cells can result in increased expression of some target genes. Because of the correlation between Suz12 binding and H3-K27 methylation, we hypothesized that the increased expression is caused by the loss of the histone methyltransferase activity after depletion of Suz12 from cells. To test this hypothesis, we examined the binding pattern of Ezh2 and the status of H3-K27 methylation on five target promoters after siRNA-mediated removal of Suz12 from SW480 cells. We incubated cells with Suz12 siRNAs for $6 \mathrm{~d}$, and then we performed ChIP assays using antibodies to Suz12, Ezh2, trimethyl H3-K27, and IgG as a control. A 6-d treatment was performed to allow for a greater loss of the methylated histones from the target promoters. As expected, we found that depletion of Suz12 from cells decreases the binding of Suz12 to the tested promoters (Fig. 8, cf. lanes 1 and 7). Interestingly, depletion of Suz12 from cells also resulted in a dramatic decrease of Ezh2 binding and H3-K27 methylation at the Suz12 target promoters. (Fig. 8, cf. lanes 2 and 8, and lanes 3 and 9). These results demonstrate that repression of the identified Suz12 target promoters is associated with methylation of H3-K27. Also, these data show that the decreased expression of Suz12 in cultured cells disrupts the function of the PRC2/3 complexes by preventing their recruitment to target promoters.

\section{Discussion}

Identification of mammalian PcG target genes has remained elusive for two main reasons. First, the majority of the previous PcG studies focused mostly on the bio-

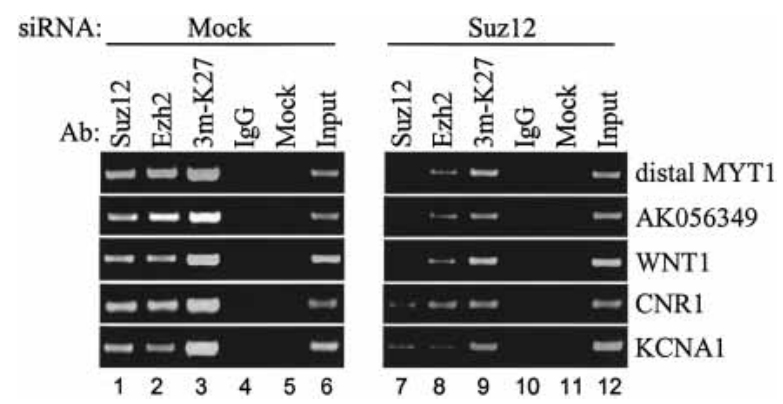

Figure 8. Methylation of H3-K27 at the Suz12 target promoters is due to Suz12-mediated recruitment of the methyltransferase Ezh2. ChIP experiments were performed using antibodies to Suz12 (lanes 1,6), Ezh2 (lanes 2,7), trimethyl H3-K27 (lanes 3,8 ), and a control IgG (lanes 4,9) in SW480 cells that were incubated with siSuz12 smart-pool or no siRNA (mock). For the siRNA transfections, cells were incubated with the indicated siRNA for $72 \mathrm{~h}$, then replated and transfected for another $72 \mathrm{~h}$. The immunoprecipitated chromatin was analyzed by PCR using primers specific to the promoters of the genes indicated. An equal amount of DNA from both treatments was monitored using PCR as shown by the similar intensity of the two input signals (cf. lanes 6 and 12). chemical purification and in vitro characterization of the activities of the PcG complexes and second, the lack of DNA-binding domains within PcG proteins makes the search for their target loci difficult. In this present study, we have identified the first known direct target genes of mammalian PcG complexes. To do so, we first used RNAi to identify genes deregulated by the loss of Suz12 protein in colon cancer cells. Next, we showed that Suz12 binds directly to the promoter of one of these genes (MYT1). We also showed that other members of the PRC2/3 complexes colocalize with Suz12 at the MYT1 promoter. Most importantly, we demonstrated that recruitment of Suz12, Ezh2, and Eed to the MYT1 promoter correlates with methylation of H3-K27. To demonstrate that this silencing mechanism is not unique to MYT1, we identified other Suz12 target genes using a ChIP assay coupled to a CpG island microarray. Similarly to MYT1, the other target promoters of Suz12 are bound by the PRC2/3 components and are characterized by H3-K27 methylation. Thus, the first identified human PcG target genes all appear to be regulated by the histone methylase activity of the PRC complexes.

The Suz12 target gene MYT1 was originally cloned from a human brain cDNA library on the basis of its ability to bind cis-regulatory elements of the glia-specific myelin proteolipid protein $(P L P)$ gene and is suggested to be the prototype of the C2HC-type zinc finger protein family (Kim and Hudson 1992). More recently, the Xenopus ortholog of MYT1 (X-MYT1) was identified as a transcriptional activator because it could induce expression of an $\mathrm{N}$-tubulin promoter reporter construct in transient transfection assays (Bellefroid et al. 1996). In the same study, the authors demonstrated that dominantnegative forms of X-MYT1 inhibited normal neurogenesis, suggesting that X-MYT1 is essential for inducing neuronal differentiation. Intriguingly, a recent report showed that the Xenopus ortholog of Ezh2 (XEZ) is expressed exclusively in the anterior neural plate during early Xenopus embryogenesis and postulated that $X E Z$ might be involved in delaying anterior neuronal differentiation (Barnett et al. 2001). Based on our findings, it is possible that Ezh2 delays neuronal differentiation, via the PRC2/3 complexes, by repressing the activity of the MYT1 gene. Besides MYT1, we have identified four additional promoters as being robustly bound by components of the PRC2/3 complexes; each of these promoters is also characterized by high levels of H3-K27. Although a link between components of the PRC2/3 complexes and Wnt1, the cannabinoid receptor (CNR1), or the potassium channel KCNA1 have not been previously reported, it is intriguing to note that these mRNAs are expressed at very low levels in most human tissues (see http://expression.gnf.org), suggesting that they may be generally silenced by the PRC complexes. In support of this hypothesis, we have shown that some of these target genes are bound by PRC2/3 components in other cell lines, such as the human MCF7 and mouse F9 (data not shown).

Previous studies have used mRNA expression profiling to identify Ezh2 target genes. In one study, gene ex- 
pression was monitored in cells that lacked Ezh2 (Bracken et al. 2003), whereas in the other study (Varambally et al. 2002) the analysis was performed in cells that overexpressed Ezh2. The genes identified in these previous studies do not overlap with the PRC2/3 target genes that we have now identified. It is possible that many, if not all, of the previously identified genes are indirectly regulated by Ezh2. It is also possible that the distinct sets of identified genes might be the result of using different cell types and/or different DNA microarrays. Therefore, our future studies will aim at the identification of a large set of mammalian PRC target genes, using various different normal and cancer cell types.

Although we have described the first known direct target genes of a human PRC complex, identifying a larger set of targets is essential to better understand the mechanisms that mediate the functions of Polycomb complexes. For example, it is unclear if all Suz12 and/or Ezh2 target genes will be regulated by the PRC2/3 complexes or if different Eed isoforms (and thus different PRC complexes) will predominate in different cell or tumor types. Therefore, it remains possible that methylation of histone $\mathrm{H} 1$, but not histone $\mathrm{H} 3$, will occur at certain PRC target genes. Also, it is possible that binding of components of the PRC2/3 complexes may sometimes lead to activation, rather than repression of gene expression. A PcG protein that has both activation and repression functions is now classified in the Enhancer of Trithorax and Polycomb (ETP) group (for review, see Brock and van Lohuizen 2001). Previous studies have shown that loss of expression of Ezh2 in Drosophila results in both increased and decreased gene expression (LaJeunesse and Shearn 1996). Similar findings were presented in another study in which siRNA-mediated depletion of Ezh2 or Eed in mammalian cells resulted in decreased expression of several genes (Bracken et al. 2003). Unfortunately, the previous studies did not distinguish between direct and indirect regulation by mammalian Ezh2 or Eed. However, our findings provide support for the hypothesis that components of the PRC2/3 complex can directly function as activators. Using ChIP, we showed that Suz12 and Ezh2 are recruited to the promoters of some genes (i.e., SYBL1) that are down-regulated upon Suz12 depletion from cells (Fig. 6; data not shown), suggesting that the PRC complexes normally activate the expression of these genes. Future studies should determine the mechanism of transcriptional activation mediated by the PRC complexes.

Finally, it is still unclear how the PRC complexes are directed to their target loci. Because no site-specific DNA-binding factor has been purified in the PRC complexes, it is not yet possible to identify a PRC target promoter by sequence inspection. In fact, even identifying a gene as being regulated by a PRC complex does not provide information as to exactly where the complex is recruited because the PRC-specific element (i.e., the PRE) might be located at a distance from the start of transcription. For example, we found that Suz12 was bound at -4000 in some promoters and at +1 in others. Interestingly, we also showed that many Suz12 target genes had multiple binding sites. In summary, this current study, which has identified the first set of human PRC2/3 target genes and demonstrated the utility of custom oligonucleotide arrays in presenting a detailed profiling of the binding of PRC components to large regions of target promoters, provides a significant step toward the goal of defining a mammalian PRE. Once we have identified a large number of direct target genes, we will use the information derived from the analysis of the custom arrays to search for common sequence elements in small, defined regions of the PRC target promoters.

\section{Materials and methods}

\section{Antibody production}

Using PCR, a 366-bp fragment from the $\mathrm{N}$ terminus and a 318 bp fragment from the $\mathrm{C}$ terminus of Suz12 ORF were generated and cloned into the Pet15b expression vector (catalog no. 69661, Novagen). The sequence of the primers can be found in Supplementary Table S3. The KIAA0160 (alternative name for Suz12) human cDNA clone, a gift from Takahiro Nagase (Kazusa DNA Research Institute, Osaka, Japan) was used as a template. Protein expression and purification were performed using the Bugbuster His-bind Purification Kit (catalog no. 70793, Novagen) according to the manufacturer's instructions. To prepare the Suz12 immunogen for rabbit injections, the N- and C-terminal protein fragments were combined in equal amounts to a final concentration of $1 \mu \mathrm{g} / \mu \mathrm{L}$. Rabbit immunizations and antibody purifications were performed at Covance Inc. The Suz12 antibody is now available at Abcam, Inc. (catalog no. ab12201).

\section{Plasmid constructs and transient transfections}

To prepare a construct expressing HA-tagged Suz12, primers containing EcoRI sites on their 5 '-ends were used in PCR to generate full-length cDNA of Suz12 (2219 bp). The KIAA0160 human cDNA clone was used as a template. The sequence of the primers can be found in Supplementary Table S3. The PCR product was digested with EcoRI and ligated into the EcoRI site of the pCMV-HA vector (Clontech). Bacterial colonies were screened for inserts and sequenced at the University of Wisconsin Biotechnology Sequencing Facility to ensure proper sequence and orientation. For transient transfections, SW480 human colon cancer cells were cultured in McCoy's 5A medium (Invitrogen) supplemented with 10\% fetal bovine serum (NovaTech) and 1\% Penicillin/Streptomycin (Invitrogen), and incubated at $37^{\circ} \mathrm{C}$ in a humidified $5 \% \mathrm{CO}_{2}$ incubator. One day before the transfection, SW480 cells were plated at $2 \times 10^{5}$ cells per $60-\mathrm{mm}$ dish. The cells were transfected with $2 \mu \mathrm{g}$ of the HA-Suz12-expressing construct (pCMV-HA-Suz12) or $2 \mu \mathrm{g}$ of the control construct (pCMV-HA-empty) using the FuGENE 6 transfection reagent (Roche) according to the manufacturer's protocol. Cells were harvested $48 \mathrm{~h}$ after transfection and were subjected to immunoblot analysis.

\section{Immunoblot analysis}

Normal and diseased human colon tissues obtained from the Cooperative Human Tissue Network (CHTN) were minced into small pieces using a razor blade. About $300 \mathrm{mg}$ of minced tissue was resuspended in $3 \mathrm{~mL}$ of $1 \times$ PBS buffer and disaggregated using a Medimachine (catalog no. 340587, Becton Dickinson) to achieve single cells. The cells were then diluted in a $2 \times$ volume 
of whole-cell lysis buffer $(0.1 \mathrm{M}$ HEPES, $0.5 \mathrm{M} \mathrm{KCl}, 5 \mathrm{mM}$ $\mathrm{MgCl}_{2}, 0.5 \mathrm{mM}$ EDTA, 35\% glycerol, $5 \mathrm{mM} \mathrm{NaF}, 1 \mathrm{mM}$ DTT, $1 \mathrm{mM}$ PMSF) and were alternately frozen and thawed three times in liquid nitrogen to rupture the cell membranes. The samples were incubated for $30 \mathrm{~min}$ on ice to lyse the nuclei and then centrifuged at $4000 \times \mathrm{g}$ for $5 \mathrm{~min}$ to pellet the cell membranes. The protein content of the resulting supernatant was quantitated using a standard Bradford assay. Equal amounts of protein $(20-40 \mu \mathrm{g})$ from the tumor and normal tissue samples of each patient were subjected to standard Western analysis. In the case of the RNAi experiments (see below) and the transient transfections (see above), $5 \times 10^{5}$ cells were harvested from each treatment and examined by a standard Western analysis. Antibodies used for probing included anti-Suz12 (described above), anti-Lamin A/C (sc-7292, Santa Cruz Biotechnology), anti-HA (catalog no. MMS-101R, Covance Research Products), and antiactin (sc-1615, Santa Cruz Biotechnology).

\section{RNA interference}

Two different 21-nt duplex siRNAs for Suz12 (target sequences of 5'-CCCGGAAATTTCCCGTCCC-3' and 5'-GAGATGACCT GCATTGCCC-3', custom orders), Suz12 smart-pool (M0069570050) and control siRNAs for Lamin A/C (target sequence 5' -CTG GACTTCCAGAAGAACA-3', D0010000105) or GFP (target sequence 5'-GGCTACGTCCAGGAGCGCACC-3', D0013000120) were synthesized by Dharmacon. For the immunoblot analysis (see above) and RNA extraction (see below), SW480 and HT29 human colon cancer cells were plated at $2 \times 10^{5}$ cells per well in a 12-well plate. Twenty-four hours after plating, the cells were transfected either with both Suz12 siRNA duplexes together (100 nmoles each) or with each control siRNA (200 nmoles each) using oligofectamine (Invitrogen) or trans-IT TKO (Mirus) according to the manufacturers' instructions. At various time points after transfection, the cells were treated with trypsin and harvested for immunoblot analysis, or RNA preparation. For the RNAi-ChIP experiments, SW480 cells were plated at a density of $1.2 \times 10^{6}$ cells per $100-\mathrm{mm}$ dish, and after $24 \mathrm{~h}$ they were transfected with $100 \mathrm{~nm}$ of Suz12 smart-pool using Lipofectamine 2000 (Invitrogen) according to the manufacturer's instructions. The cells were incubated with the Suz12 smart-pool for $72 \mathrm{~h}$ and then were harvested and replated for another $72 \mathrm{~h}$ of transfection with $100 \mathrm{~nm}$ of Suz12 smart-pool. At the end of the second 72-h incubation, the cells were harvested and used in ChIP assays as described below.

\section{RT-PCR analysis}

Total RNA was prepared from $1 \times 10^{6}$ SW480 cells of each indicated treatment using the RNeasy kit (QIAGEN) as described by the manufacturer. For each RT-PCR reaction, 100 ng of RNA was used, and the amplifications were performed as described previously (Graveel et al. 2001). A list of all the primer sequences used in RT-PCR is available as Supplementary Table S3. All the primers used in this study were obtained from the University of Wisconsin Biotechnology Center.

\section{RNA microarray analysis}

Total RNA was extracted from cells transfected with either Suz12 siRNAs or control GFP siRNA as described above. For each treatment, RNA was prepared from three independent experiments and was combined into one sample to reduce the experimental variation. Targets for microarray hybridization were generated from the RNA according to the supplier's instructions (Affymetrix). The human U133A gene chip (Af- fymetrix) was used for gene expression profiling, which represents $\sim 33,000$ transcripts. Four U133A arrays were used for this analysis; two arrays were hybridized with cRNA obtained from cells treated with siRNAs to Suz12, and two arrays were hybridized with cRNA obtained from cells treated with siRNA to GFP. Hybridization, washing, scanning, and analysis of gene chips were performed at the University of Wisconsin Gene Expression Center according to the manufacturer's instructions. Expression levels were analyzed by the statistical algorithm in the Microarray Analysis Suite (MAS) 5.0 software (Affymetrix) using the default parameters. The data from the GFP siRNA treatment were used as a baseline expression for comparison with the Suz12 siRNA-treated sample.

\section{ChIPs}

Formaldehyde cross-linking and ChIP in SW480 cells were performed as described before (Weinmann et al. 2001) with the following exceptions. Precleared chromatin from $2 \times 10^{6}$ cells was used for each ChIP sample. After reversing the cross-links, each individual ChIP sample was purified using the Qiaquick PCR purification kit (QIAGEN), and samples were eluted with $30 \mu \mathrm{L}$ of elution buffer. The antibodies used in the ChIP assays included purified Suz12 antibody (see above), Ezh2, Eed-M26, trimethyl H3-K27, trimethyl H3-K9 (ab8898, Abcam Inc.), RNA Polymerase II (sc-899, Santa Cruz Biotechnology), histone H3 (ab1791, Abcam Inc.), rabbit anti-goat (catalog no. 55335, ICN Pharmaceuticals; used as an IgG control), and rabbit preimmune serum (Covance; used as a control in CpG-island array experiments). All antibodies were used at $1 \mu \mathrm{g}$ per IP except the Suz12 antibody, which was used at $3 \mu \mathrm{g}$ per IP. For the immunogen IP control (experiments shown in Fig. 4), Suz12 antibody was mixed with a 10 -fold excess by weight of immunogen (bacterially expressed Suz12 protein fragments) and incubated with rotation at $4^{\circ} \mathrm{C}$ overnight prior to use in the ChIP assay. A detailed protocol of the ChIP procedure and the sequences of all the primers used in PCR can be found in Supplementary Table S3.

\section{$L M-P C R$}

The generation of amplicons from individual ChIP samples was adapted from Ren et al. (2000). A detailed protocol can be found in Oberley et al. (2004) or at http://mcardle.oncology.wisc.edu/ farnham.

\section{Oligonucleotide promoter array analysis}

Oligonucleotide promoter array analysis was performed by NimbleGen Systems as part of a Chromatin Immunoprecipitation (ChIP) Array Service. The custom arrays, each having 362,890 60-nt oligonucleotides, contained probes that tiled through $5 \mathrm{~kb}$ of the promoter regions of the selected genes. The probes represented both the forward and reverse strands, were spaced every 15 bases, and were printed at five (reverse strand) or six (forward strand) random locations on the array. For labeling, hybridization, and data analysis, refer to the Supplemental Material.

\section{CpG-island microarray analysis}

For detailed protocols on these steps, see Oberley et al. (2004) or http://mcardle.oncology.wisc.edu/farnham. The Cy5 dye was coupled to the Suz12 (or the preimmune) amplicon, and Cy3 dye was coupled to the input reference amplicon, using standard methods. Labeled chromatin was hybridized with human CpGisland microarrays obtained from the Ontario University Health 
Network (http://www.microarrays.ca). Hybridizations were performed as described previously (Oberley et al. 2003). The hybridized microarrays were analyzed using the Genepix Pro 4.1 (Axon Instruments) software package. This provided a set of raw values for each feature on each array. To identify clones that are selectively enriched during ChIP relative to the starting population, the Cy5 and Cy3 channels were normalized across the entire array, by taking the ratio of the medians for all features and normalizing them to unity. After normalization, features of poor intensity $(<500)$ and those that had obvious blemishes were manually flagged and removed from the putative positive list. For all quality features, a ratio was generated for each feature that was the intensity in the Cy5 channel minus background divided by the intensity in the $\mathrm{Cy} 3$ channel minus background. Features with Suz12/Input intensity ratios above 3 and PI/Input intensity ratios below 1.3 were selected for further analysis.

\section{Acknowledgments}

We thank Takahiro Nagase for providing the KIAA0160 cDNA clone and Arie Otte for providing the anti-EED (M26) antibody; Sandra Splinter, Wayne Davis, Mike Singer, and Todd Richmond for assistance with the microarray analyses; and Matthew J. Oberley for helpful discussions. We also thank the Cooperative Human Tissue Network (CHTN) for providing samples of human colon tissues. This work was supported by a DOD grant (BC020760) to P.J.F., and by an NIH grant (GM37120) and the Howard Hughes Medical Institute to D.R.

The publication costs of this article were defrayed in part by payment of page charges. This article must therefore be hereby marked "advertisement" in accordance with 18 USC section 1734 solely to indicate this fact.

\section{References}

Barnett, M.W., Seville, R.A., Nijjar, S., Old, R.W., and Jones, E.A. 2001. Xenopus Enhancer of zeste (XEZ); an anteriorly restricted polycomb gene with a role in neural patterning. Mech. Dev. 102: 157-167.

Bellefroid, E.J., Bourguignon, C., Hollemann, T., Ma, Q., Anderson, D.J., Kintner, C., and Pieler, T. 1996. X-MyT1, a Xenopus C2HC-type zinc finger protein with a regulatory function in neuronal differentiation. Cell 87: 1191-1202.

Bienz, M. and Clevers, H. 2000. Linking colorectal cancer to Wnt signaling. Cell 103: 311-320.

Bloyer, S., Cavalli, G., Brock, H.W., and Dura, J.M. 2003. Identification and characterization of polyhomeotic PREs and TREs. Dev. Biol. 261: 426-442.

Bracken, A.P., Pasini, D., Capra, M., Prosperini, E., Colli, E., and Helin, K. 2003. EZH2 is downstream of the pRB-E2F pathway, essential for proliferation and amplified in cancer. EMBO J 22: 5323-5335.

Breiling, A., Turner, B.M., Bianchi, M.E., and Orlando, V. 2001. General transcription factors bind promoters repressed by Polycomb group proteins. Nature 412: 651-655.

Brock, H.W. and van Lohuizen, M. 2001. The Polycomb groupNo longer an exclusive club? Curr. Opin. Genet. Dev. 11: 175-181.

Cao, R., Wang, L., Xia, L., Erdjument-Bromage, H., Tempst, P., Jones, R.S., and Zhang, Y. 2002. Role of histone H3 lysine 27 methylation in Polycomb-group silencing. Science 298: 1039-1043.

Czermin, B., Melfi, R., McCabe, D., Seitz, V., Imhof, A., and
Pirrotta, V. 2002. Drosophila enhancer of Zeste/ESC complexes have a histone $\mathrm{H} 3$ methyltransferase activity that marks chromosomal Polycomb sites. Cell 111: 185-196.

Dellino, G.I., Schwartz, Y.B., Farkas, G., McCabe, D., Elgin, S.C.R., and Pirrotta, V. 2004. Polycomb silencing blocks transcription initiation. Mol. Cell. 13: 887-893.

Graveel, C.R., Jatkoe, T., Madore, S.J., Holt, A.L., and Farnham, P.J. 2001. Expression profiling and identification of novel genes in hapatocellular carcinomas. Oncogene 20: 27042712.

Jacobs, J.J. and van Lohuizen, M. 1999. Cellular memory of transcriptional states by Polycomb-group proteins. Semin. Cell Dev. Biol. 10: 227-235.

. 2002. Polycomb repression: From cellular memory to cellular proliferation and cancer. Biochim. Biophys. Acta 1602: 151-161.

Jurgens, G. 1985. A group of genes controlling the spatial expression of the bithorax complex in Drosophila. Nature 316: 153-155.

Kim, J.G. and Hudson, L.D. 1992. Novel member of the zinc finger superfamily: A C2-HC finger that recognizes a gliaspecific gene. Mol. Cell. Biol. 12: 5632-5639.

Kirmizis, A., Bartley, S.M., and Farnham, P.J. 2003. Identification of the Polycomb group protein $\mathrm{SU}(\mathrm{Z}) 12$ as a potential molecular target for human cancer therapy. Mol. Cancer Ther. 2: 113-121.

Kleer, C.G., Cao, Q., Varambally, S., Shen, R., Ota, I., Tomlins, S.A., Ghosh, D., Sewalt, R.G., Otte, A.P., Hayes, D.F., et al. 2003. EZH2 is a marker of aggressive breast cancer and promotes neoplastic transformation of breast epithelial cells. Proc. Natl. Acad. Sci. 100: 11606-11611.

Koontz, J.I., Soreng, A.L., Nucci, M., Kuo, F.C., Pauwels, P., van den Berghe, H., Dal Cin, P., Fletcher, J.A., and Sklar, J. 2001. Frequent fusion of the JAZF1 and JJAZ1 genes in endometrial stromal tumors. Proc. Natl. Acad. Sci. 98: 6348-6353.

Kuzmichev, A., Nishioka, K., Erdjument-Bromage, H., Tempst, P., and Reinberg, D. 2002. Histone methyltransferase activity associated with a human multiprotein complex containing the Enhancer of zeste protein. Genes \& Dev. 16: 2893 2905.

Kuzmichev, A., Jenuwein, T., Tempst, P., and Reinberg, D. 2004. Different Ezh2-containing complexes target methylation of histone $\mathrm{H} 1$ or nucleosomal histone H3. Mol. Cell 14: 183-193.

LaJeunesse, D. and Shearn, A. 1996. E(z): A polycomb group gene or a trithorax group gene? Development 122: 2189-2197.

Muller, J., Hart, C.M., Francis, N.J., Vargas, M.L., Sengupta, A., Wild, B., Miller, E.L., O'Connor, M.B., Kingston, R.E., and Simon, J.A. 2002. Histone methyltransferase activity of a Drosophila Polycomb group repressor complex. Cell 111: 197-208.

Oberley, M.J., Inman, D., and Farnham, P.J. 2003. E2F6 negatively regulates BRCA1 in human cancer cells without methylation of histone $\mathrm{H} 3$ on lysine 9. J. Biol. Chem. 278: 42466-42476.

Oberley, M.J., Tsao, J., Yau, P., and Farnham, P.J. 2004. High throughput screening of chromatin immunoprecipitates using CpG island microarrays. Methods Enzymol. 376:316335.

Orlando, V. 2003. Polycomb, epigenomes, and control of cell identity. Cell 112: 599-606.

Orlando, V., Jane, E.P., Chinwalla, V., Harte, P.J., and Paro, R. 1998. Binding of Trithorax and Polycomb proteins to the bithorax complex: Dynamic changes during early Drosophila embryogenesis. EMBO J. 17: 5141-5150.

Pasini, D., Bracken, A.P., and Helin, K. 2004. Polycomb group 
proteins in cell cycle progression and cancer. Cell Cycle 3: 22-26.

Pirrotta, V., Poux, S., Melfi, R., and Pilyugin, M. 2003. Assembly of Polycomb complexes and silencing mechanisms. Genetica 117: 191-197.

Poux, S., Melfi, R., and Pirrotta, V. 2001. Establishment of Polycomb silencing requires a transient interaction between PC and ESC. Genes \& Dev. 15: 2509-2514.

Ren, B., Robert, F., Wyrick, J.J., Aparicio, O., Jennings, E.G., Simon, I., Zeitlinger, J., Schreiber, J., Hannett, N., Kanin, E., et al. 2000. Genome-wide location and function of DNA binding proteins. Science 290: 2306-2309.

Saurin, A.J., Shao, Z., Erdjument-Bromage, H., Tempst, P., and Kingston, R.E. 2001. A Drosophila Polycomb group complex includes Zeste and dTAFII proteins. Nature 412: 655-660.

Shao, Z., Raible, F., Mollaaghababa, R., Guyon, J.R., Wu, C.T., Bender, W., and Kingston, R.E. 1999. Stabilization of chromatin structure by PRC1, a Polycomb complex. Cell 98: 3746.

Simon, J.A. 2003. Polycomb group proteins. Curr. Biol. 13: R79R80.

Simon, J., Chiang, A., Bender, W., Shimell, M.J., and O'Connor, M. 1993. Elements of the Drosophila bithorax complex that mediate repression by Polycomb group products. Dev. Biol. 158: 131-144.

Tie, F., Prasad-Sinha, J., Birve, A., Rasmuson-Lestander, A., and Harte, P.J. 2003. A 1-megadalton ESC/E(Z) complex from Drosophila that contains polycomblike and RPD3. Mol. Cell. Biol. 23: 3352-3362.

Varambally, S., Dhanasekaran, S.M., Zhou, M., Barrette, T.R., Kumar-Sinha, C., Sanda, M.G., Ghosh, D., Pienta, K.J., Sewalt, R.G., Otte, A.P., et al. 2002. The polycomb group protein EZH2 is involved in progression of prostate cancer. $\mathrm{Na}$ ture 419: 624-629.

Weinmann, A.S., Bartley, S.M., Zhang, M.Q., Zhang, T., and Farnham, P.J. 2001. The use of chromatin immunoprecipitation to clone novel E2F target promoters. Mol. Cell. Biol. 21: 6820-6832.

Weinmann, A.S., Yan, P.S., Oberley, M.J., Huang, T.H.-M., and Farnham, P.J. 2002. Isolating human transcription factor targets by coupling chromatin immunoprecipitation and CpG island microarray analysis. Genes \& Dev. 16: 235-244.

Wells, J., Yan, P.S., Cechvala, M., Huang, T., and Farnham, P.J. 2003. Identification of novel $\mathrm{pRb}$ binding sites using CpG microarrays suggests that E2F recruits $\mathrm{pRb}$ to specific genomic sties during S phase. Oncogene 22: 1445-1460. 


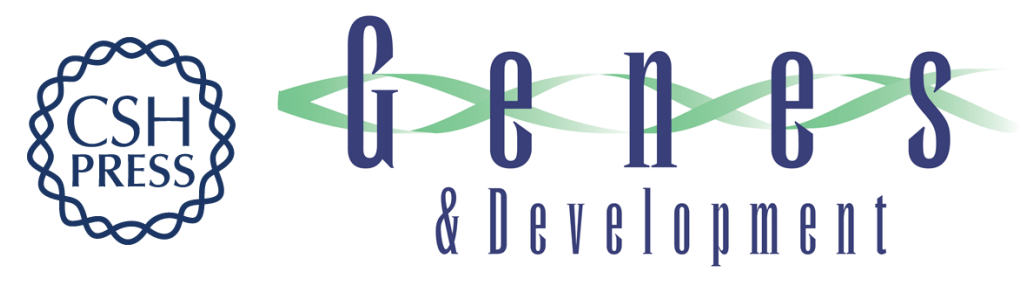

\section{Silencing of human polycomb target genes is associated with methylation of histone H3 Lys 27}

Antonis Kirmizis, Stephanie M. Bartley, Andrei Kuzmichev, et al.

Genes Dev. 2004, 18:

Access the most recent version at doi:10.1101/gad.1200204

Supplemental http://genesdev.cshlp.org/content/suppl/2004/06/14/18.13.1592.DC1
Material

References This article cites 39 articles, 15 of which can be accessed free at:

http://genesdev.cshlp.org/content/18/13/1592.full.html\#ref-list-1

License

Email Alerting

Receive free email alerts when new articles cite this article - sign up in the box at the top

Service

right corner of the article or click here.

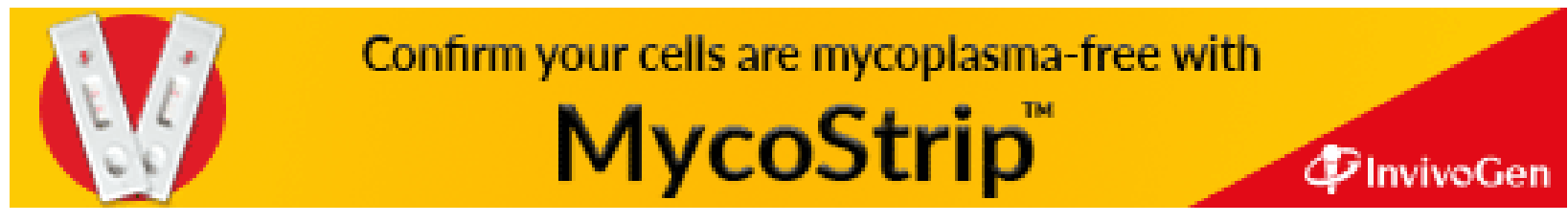

